\title{
Quantum State Reconstruction of Many Body System Based on Complete Set of Quantum Correlations Reduced by Symmetry
}

\author{
X.F. Liu ${ }^{1, \text { 田 }}$ and C.P. $\operatorname{Sun}^{2, \dagger}$ \\ ${ }^{1}$ Department of Mathematics, Peking University, Beijing, 100871, China \\ ${ }^{2}$ Institute of Theoretical Physics, Chinese Academy of Sciences, Beijing, 100080, China
}

\begin{abstract}
We propose and study a universal approach for the reconstruction of quantum states of many body systems from symmetry analysis. The concept of minimal complete set of quantum correlation functions (MCSQCF) is introduced to describe the state reconstruction. As an experimentally feasible physical object, the MCSQCF is mathematically defined through the minimal complete subspace of observables determined by the symmetry of quantum states under consideration. An example with broken symmetry is analyzed in detail to illustrate the idea.
\end{abstract}

PACS numbers: 03.65.Fd, 02.20.Qs, 05.50.+q,03.67.Mn

Introduction: The concept of quantum state is fundamental in quantum physics. Quantum state can provide us with a complete knowledge of the considered system to predict the possible results of any measurement on the system [1]. But the puzzling property of quantum coherence, which is intrinsic to quantum state, imposes insurmountable limitations on our ability to fully reconstruct quantum state of a single quantum system by devising a complete set of measurements on the system [2].

Actually, we cannot make a measurement on a single quantum system without back-action and the noncloning theorem [3] forbids us from producing an exact copy of some quantum system in an unknown state. But on the other hand, if identical copies of quantum system, or an ensemble, is initially prepared in the same quantum state, it is possible to estimate the unknown state of the quantum system by carrying out appropriate measurements on each copy [4]. This is just the idea of quantum tomography, which has recently become a fashion in quantum information physics, motivating many theoretical and experimental investigations [5].

We notice that most works on quantum tomography or other quantum state reconstruction methods focus on continuous variable system and single qubit system. In this letter we will deal with the many particle case, which possesses distinguished features such as quantum entanglements [6]. It has become well known that quantum correlation and the corresponding Green function are important conceptual tools to probe the non-local nature of many body system. On the other hand, in the study of quantum information, quantum entanglement turns out to be a crucial concept and thus becomes a fundamental element in the research about the nonlocality of quantum system. Hence, it is doubtlessly important and meaningful to probe the possible connection between quantum entanglement and quantum correlation [7]. Along this line, some progress has been made. For example, for the spin chain system with the symmetry of $S O(2)$, a connection has been revealed between the concurrence, which is a measure of quantum entanglement, and the correlation functions of first order and second order, which is supposed to reflect the property of correlation [8]. This connection suggests a possible connection between quantum entanglement and quantum phase transition 9, 10, 11 and prompts us to introduce in this letter a general framework for studying such connection.

Our starting point is the basic observation that complete information about quantum state of many body system can be obtained from the expectation values of properly chosen single body observables and their correlation functions of various orders 12. And our idea is to reduce the number of times of measurement needed to determine a quantum state by making full use of the symmetry that the state is supposed to possess. The main purpose of this letter is to make clear the mechanism of this reduction and present interesting examples to illustrate it. To this end we will introduce the minimal complete subspace of observables (MCSO) associated with a symmetry group, which mathematically determines the quantum state with this symmetry, and the minimal complete set of quantum correlation functions (MCSQCF) associated with the MCSO, which determines the quantum state physically in some sense.

State Reconstruction Based on Correlation Functions: Throughout this letter, let $V=\otimes_{j=1}^{m} W_{j} \triangleq W_{1} \otimes W_{2} \otimes$ $\cdots \otimes W_{m}$ be the Hilbert space of a many spin system. Here the $W_{i}$ 's stand for the state space $W$ of a spin system. For an arbitrary finite dimensional Hilbert space $S$ we denote by $\mathcal{A}(S)$ the set of Hermitian operators on $S$. It is well known that $\mathcal{A}(S)$ is an Euclidean space with the inner product $\langle$,$\rangle defined as \langle A, B\rangle=\operatorname{tr}(A B), \forall A, B \in$ $\mathcal{A}(S)$, where tr is the trace over $W$.

Now let $\rho \in \mathcal{A}(V)$ be a density operator and $\left\{A_{i}\right\}$ a basis of $\mathcal{A}(V)$. Then $\rho$ can be written as a linear combination of $A_{i}$ 's: $\rho=\sum_{i} c_{i} A_{i}$. We notice that the coefficients $c_{i}$ 's are determined by the expectation values $\left\{\operatorname{tr}\left(\rho A_{i}\right)\right\}$ of the observable $A_{i}$ in the state $\rho$. In particular, if $\left\{A_{i}\right\}$ is an orthonormal basis then we have $c_{i}=\operatorname{tr} \rho A_{i}$. Obviously, from mathematical point of view it is trivially true that $\rho$ is completely determined by $\left\{\left\langle\rho, A_{i}\right\rangle\right\}$. But phys- 
ically it means the possibility of determining a quantum state by measuring some properly chosen observables.

To be precise, let us choose a basis $\left\{O_{i}\right\}$ of $\mathcal{A}(W)$. The $O_{i}$ 's can be understood as observables of single particle system. Then $\left\{\otimes_{k=1}^{m} O_{i_{k}}\right\}$ is a basis of $\mathcal{A}(V)$, where each $O_{i_{j}}$ belongs to $\left\{O_{i}\right\}$. Hence we can identify the state $\rho$ by the quantities

$$
\operatorname{tr}\left(\rho \otimes_{k=1}^{m} O_{i_{k}}\right) \triangleq\left\langle\otimes_{k=1}^{m} O_{i_{k}}\right\rangle_{\rho},
$$

which are just correlation functions of the considered system.

For spin $1 / 2$ system interesting things happen [7, 8]. In this case we can take $\left\{O_{i}\right\}$ to be $\left\{\sigma_{0}, \sigma_{1}, \sigma_{2}, \sigma_{3}\right\}$, where $\sigma_{0}$ is the identity operator and $\sigma_{1}, \sigma_{2}, \sigma_{3}$ are the Pauli operators $\sigma_{x}, \sigma_{y}, \sigma_{z}$. Let us introduce the standard correlation functions $G_{i_{1} i_{2} \cdots i_{n}}^{\rho} \triangleq\left\langle\otimes_{k=1}^{n} \sigma_{i_{k}}\right\rangle_{\rho}$, associated with the state $\rho$. Clearly, from both theoretical and experimental points of view standard correlation functions are accessible physical quantities. According to the discussion above, for spin $1 / 2$ system a state is determined by these ideal quantities.

We are not so lucky when we consider higher spin system. The main reason is, when the dimension of $W$ is larger than 2 it is impossible to span $\mathcal{A}(W)$ with just the spin operators and identity operators. This forces us to consider the correlation functions not related to the spin operators in a direct way. From experimental point of view such correlation functions are less physical than the standard correlation functions. But from theoretical point of view there is no essential difference between them.

Now a natural question is: how many correlation functions are needed to determine a quantum state? Obviously, if we have no knowledge about the state, then the number of correlation functions needed is exactly the dimension of $\mathcal{A}(V)$. But if we are sure that the state possesses some symmetry, then it turns out that the number might be greatly reduced. Let us elucidate this point in detail as follows.

Reduction to the Minimal Complete Subspace of Observables by Symmetry: Now, we discuss symmetry of density operator and the role symmetry can play in the reconstruction of density operator.

The symmetry group of the density operator $\rho \in \mathcal{A}(V)$ is defined to be the subgroup $U_{\rho}$ of $U(n)$ with the property $u \rho u^{\dagger}=\rho, \forall u \in U_{\rho}$. If $U$ is a subgroup of $U_{\rho}$ we will say that $\rho$ possesses the symmetry of $U$. In practice, it is highly possible that only partial information about the symmetry of a density operator is available. In other words, one may only be sure that some group is a subgroup of the symmetry group of a density operator. This being the case, it is desirable to know how to identify a density operator among a family of density operators whose symmetry groups contain a specific unitary group $U$ (a subgroup of $U(n)$ by definition). This is the idea underlying the following discussion.
A symmetry of $U$ determines a family $O_{U}$ of observables in the following way: $A \in O_{U}$ if and only if $u A u^{\dagger}=A, \forall u \in U$. By definition, if $A \in O_{U}$ then its symmetry group contains $U$ as a subgroup and vice versa. Thus $\rho$ belongs to $O_{U}$ if and only if it possesses the symmetry of $U$. Clearly, $O_{U}$ is a subspace of $\mathcal{A}(V)$. We call it the MCSO associated with $U$.

As $\mathcal{A}(V)$ is an inner product space it can be decomposed as $\mathcal{A}(V)=O_{U} \oplus O_{U}^{\perp}$, where $O_{U}^{\perp}$ is the orthonormal complement to $O_{U}$. Let $\left\{A_{i}\right\}$ be an arbitrary basis of $O_{U}$. Then each element $A$ of $O_{U}$ is uniquely determined by the quantities $\left\{\left\langle A, A_{i}\right\rangle\right\}$. This justifies calling $O_{U}$ complete. Consequently, if $\rho$ possesses the symmetry of $U$ then the number of measurements needed to determine $\rho$ is just the dimension of the MCSO associated with $U$.

Next let us determine the structure of $O_{U}$.

Proposition 1. If $U$ is connected, then $i O_{U}$ is the centralizer of the Lie algebra of $U$ in $u(n)$, the Lie algebra of $U(n)$, where $n$ is the dimension of $V$. ( Here $i=\sqrt{-1}$.)

Proof. We notice that $i \mathcal{A}(V)$ is exactly the Lie algebra of $U(n)$. Let $i A$ be an arbitrary element of the Lie algebra of $U$ and $i B$ an arbitrary element of $i O_{U}$. Then we have $i B=\exp ($ it $A)(i B) \exp (-i$ t $A)$ or

$$
i B=\left(\operatorname{Ad} e^{i t A}\right)(i B)=e^{i t \operatorname{ad} A}(i B) .
$$

It follows that $i O_{U}$ is a subset of the centralizer of the Lie algebra of $U$. That they are actually identical is now a consequence of the fact that if $U$ is connected, then it can be generated by 1-parameter subgroups. This completes the proof of the proposition.

When $U$ is a 1-parameter subgroup the structure of $O_{U}$ can be given a concise description. Let us consider this case in more detail. Suppose that $U=\exp (-i t A)$ where $A \in \mathcal{A}(V)$, then according to Proposition 1, an element $B$ belongs to $O_{U}$ if and only if $[A, B]=0$. As $A$ is an Hermitian operator there exists an orthonormal basis $\gamma$ of $V$ with respect to which it has the matrix representation of block diagonal form (the meaning of notation here should be self-evident): $A=\operatorname{diag}\left\{A_{1}, A_{2}, \cdots, A_{n}\right\}$, where $A_{i}=\lambda_{i} I_{n_{i}}, \lambda_{i}$ being an eigenvalue of $A$ and $I_{n_{i}}$ is the unit matrix of rank $n_{i}, n_{i}$ being the multiplicity of $\lambda_{i}$. From this observation, the following proposition follows directly.

Proposition 2. If $U$ is connected then an element $B$ lies in $O_{U}$ if and only if its matrix representation takes the form $B=\operatorname{diag}\left\{B_{1}, B_{2}, \cdots, B_{n}\right\}$, with respect to the same basis $\gamma$ defined above, where $B_{i}$ is an $n_{i}$ by $n_{i}$ matrix.

Symmetry of Reduced Density Operators: In this section we consider symmetry of the so called reduced density operator, which is derived from the density operator after a process of taking partial trace. We are interested in the symmetry that survives such process.

Let $V=V_{1} \otimes V_{2}$ and $\rho$ a density operator in $\mathcal{A}(V)$. The reduced density operator $\operatorname{tr}_{2} \rho$ is defined to be an 
element of $\mathcal{A}\left(V_{1}\right)$ satisfying the relations $\left(x_{1},\left(\operatorname{tr}_{2} \rho\right) x_{2}\right)=$ $\sum_{i}\left(x_{1} \otimes y_{i}, \rho x_{2} \otimes y_{i}\right), \forall x_{1}, x_{2} \in V_{1}$, where $\left\{y_{i}\right\}$ is a basis of $V_{2}$. Note that we have used the same notation (, ) for the inner products in the different spaces. It is easily check that $\operatorname{tr}_{2} \rho$ is also a density operator. Now the following lemma comes as a direct consequence of the definitions. We would rather omit the proof.

Lemma 1. If $\rho$ possesses the symmetry of $U_{1} \otimes U_{2}$ then $\operatorname{tr}_{2} \rho$ possesses the symmetry of $U_{1}$.

Although the idea of studying how symmetry survives the reduction process might seem trivial, there are nontrivial examples to support it. Let us consider two models of such examples. For convenience, from now on we will use the Dirac notation.

Suppose that $A \in \mathcal{A}(V)$ has the form $A=A_{1} \otimes 1+$ $1 \otimes A_{2}$, where $A_{1} \in \mathcal{A}\left(V_{1}\right)$ and $A_{2} \in \mathcal{A}\left(V_{2}\right)$. Let $|\psi\rangle$ be a normalized eigenvector of $A$. Then it is easily seen that the density operator $\rho=|\psi\rangle\langle\psi|$ possesses the symmetry of the 1-parameter subgroup $\exp ($ it $A)$ that can be written as $\exp ($ it $A)=\exp \left(\right.$ it $\left.A_{1}\right) \otimes \exp \left(\right.$ it $\left.A_{2}\right)$. Thus the reduced density operator $\operatorname{tr}_{2} \rho$ possesses the symmetry of $\exp \left(\right.$ it $\left.A_{1}\right)$ according to Lemma 1.

In the special case that $\rho$ has the symmetry of 1 parameter subgroup there is another mechanism of passing symmetry to the reduced density operator, which is different from the mechanism shown above. Let $A$ be an Hermitian operator of the form $A=A_{1} \otimes A_{2}$ where $A_{1} \in \mathcal{A}\left(V_{1}\right)$ and $A_{2} \in \mathcal{A}\left(V_{2}\right)$ and $|\psi\rangle$ a normalized eigenvector of $A$. Then for the density operator $\rho=|\psi\rangle\langle\psi|$ we have the following result.

Lemma 2. The reduced density operator $\operatorname{tr}_{2} \rho$ possesses the symmetry of $\exp \left(i t A_{1}\right)$ if the eigenvalue corresponding to $|\psi\rangle$ is nonzero.

Proof. Let $\left\{\left|\phi_{i}\right\rangle\right\}$ be an orthonormal basis of $V_{1}$ consisting of the eigenvectors of $A_{1}$ and $\left\{\left|\varphi_{j}\right\rangle\right\}$ an orthonormal basis of $V_{2}$ consisting of the eigenvectors of $A_{2}$. Suppose that $A_{1}\left|\phi_{i}\right\rangle=\lambda_{i}\left|\phi_{i}\right\rangle$ and $A_{2}\left|\varphi_{j}\right\rangle=\mu_{j}\left|\varphi_{j}\right\rangle$. Since $|\psi\rangle$ is an eigenvector of $A$ there exist $\lambda_{k}$ and $\mu_{l}$ such that $A|\psi\rangle=\lambda_{k} \mu_{l}|\psi\rangle$. By definition, for arbitrary two elements $\left|\phi_{i_{1}}\right\rangle$ and $\left|\phi_{i_{2}}\right\rangle$ of the basis $\left\{\left|\phi_{i}\right\rangle\right\}$ we have $\left\langle\phi_{i_{1}}\left|\operatorname{tr}_{2} \rho\right| \phi_{i_{2}}\right\rangle=$ $\sum_{j}\left\langle\varphi_{j}, \phi_{i_{1}} \mid \psi\right\rangle\left\langle\psi \mid \phi_{i_{2}}, \varphi_{j}\right\rangle$, where $\left|\phi_{i_{2}}, \varphi_{j}\right\rangle=\left|\phi_{i_{2}}\right\rangle \otimes\left|\varphi_{j}\right\rangle$. It follows that $\left\langle\phi_{i_{1}}\left|\operatorname{tr}_{2} \rho\right| \phi_{i_{2}}\right\rangle=0$, unless $\lambda_{i_{1}}=\lambda_{i_{2}}$. On the other hand, we have

$$
P=e^{i t\left(\lambda_{i_{1}}-\lambda_{i_{2}}\right)}\left\langle\phi_{i_{1}}\left|\operatorname{tr}_{2} \rho\right| \phi_{i_{2}}\right\rangle .
$$

for $P \triangleq\left\langle\phi_{i_{1}}\left|\exp \left(i t A_{1}\right) \operatorname{tr}_{2} \rho \exp \left(-i t A_{1}\right)\right| \phi_{i_{2}}\right\rangle$. This just means that

$$
P=\left\{\begin{array}{cc}
\left\langle\phi_{i_{1}}\left|\operatorname{tr}_{2} \rho\right| \phi_{i_{2}}\right\rangle, & \text { if } \lambda_{i_{1}}=\lambda_{i_{2}} \\
0, & \text { otherwise. }
\end{array}\right.
$$

Thus we conclude that $\exp \left(\right.$ it $\left.A_{1}\right) \operatorname{tr}_{2} \rho \exp \left(-i t A_{1}\right)=$ $\operatorname{tr}_{2} \rho$ and complete the proof.

Minimal Complete Set of Correlation Functions: In this section the problem of reconstruction of quantum state is reduced to the problem of determination of a set of correlation functions.
Let $\left\{O_{i}\right\}$ be a basis of $\mathcal{A}(W)$. Then $\left\{\otimes_{k=1}^{m} O_{i_{k}}\right\}$ is a basis of $\mathcal{A}(V)$. We call it the measurement basis of $\mathcal{A}(V)$ associated with $\left\{O_{i}\right\}$. A subset of the measurement basis of $\mathcal{A}(V)$ is called a reconstruction basis of the MCSO associated with $\left\{O_{i}\right\}$ if each element of the MCSO can be written as a linear combination of elements in the subset and a reconstruction basis is called minimal if it does not contain any smaller reconstruction basis. Note that according to this definition elements of a reconstruction basis of the MCSO do not necessarily belong to the MCSO.

Proposition 3. There exists a unique minimal reconstruction basis of an MCSO associated with each $\left\{O_{i}\right\}$.

Proof. Choose an arbitrary basis $\beta$ of the considered MCSO and define a subset $\gamma_{1}$ of the measurement basis $\left\{\otimes_{k=1}^{m} O_{i_{k}}\right\}$ as follows: an element lies in $\gamma_{1}$ if and only if it appears as a non zero term in the expression of some element of $\beta$ in terms of $\left\{\otimes_{k=1}^{m} O_{i_{k}}\right\}$. Clearly $\gamma_{1}$ is a minimal reconstruction basis associated with $\left\{O_{i}\right\}$. We claim that it is in fact the unique minimal reconstruction basis of the considered MCSO associated with $\left\{O_{i}\right\}$.

Let $\gamma_{2}$ be another minimal reconstruction basis associated with $\left\{O_{i}\right\}$. Then each element of $\beta$ can be expressed as a linear combination of the elements of $\gamma_{2}$ or as a linear combination of the elements of $\gamma_{1}$. Thus by equating the two expressions we conclude that $\gamma_{1} \subseteq \gamma_{2}$, considering the fact that both $\gamma_{1}$ and $\gamma_{2}$ are subsets of the measurement basis $\left\{\otimes_{k=1}^{m} O_{i_{k}}\right\}$. That $\gamma_{1}$ and $\gamma_{2}$ are actually identical now follows from the minimality of $\gamma_{2}$. This completes the proof of the proposition.

We are now in a position to introduce the concept of MCSQCF. Let $O_{U}$ be an MCSO and $\left\{\otimes_{k=1}^{m} O_{j_{k}}\right\}$ the minimal reconstruction basis of $O_{U}$ associated with $\left\{O_{i}\right\}$. Please note the notational difference between $\left\{\otimes_{k=1}^{m} O_{j_{k}}\right\}$ and $\left\{\otimes_{k=1}^{m} O_{i_{k}}\right\}$. Here the former is used to denote a subset of the latter. With respect to $\left\{\otimes_{k=1}^{m} O_{i_{k}}\right\}$, the MCSQCF associated with $O_{U}$ is defined to be the set $\left\{\left\langle\otimes_{k=1}^{m} O_{j_{k}}\right\rangle_{\rho}\right\}$ of quantum correlation functions where $\rho$ stands for a density operator in $O_{U}$. The next proposition shows that each $\rho$ in $O_{U}$ is completely determined by $\left\{\left\langle\otimes_{k=1}^{m} O_{j_{k}}\right\rangle_{\rho}\right\}$ but not by any proper subset of it. This is just what "complete" and "minimal" mean in the definition of MCSQCF.

Proposition 4. A density operator $\rho$ in $O_{U}$ can be expressed in terms of the MCSQCF $\left\{\left\langle\otimes_{k=1}^{m} O_{j_{k}}\right\rangle_{\rho}\right\}$, but cannot be expressed in terms of any proper subset of $\left\{\left\langle\otimes_{k=1}^{m} O_{j_{k}}\right\rangle_{\rho}\right\}$, regardless of the basis of $V$ on which $\rho$ acts.

Proof. Let $\left\{B_{i}\right\}$ be an orthonormal basis of $O_{U}$. We then have $\rho=\sum_{i}\left\langle\rho, B_{i}\right\rangle B_{i}=\sum_{i}\left(\operatorname{tr} \rho B_{i}\right) B_{i}$, where each coefficient $\operatorname{tr} \rho B_{i}$ can be written as a linear combination of $\left\{\left\langle\otimes_{k=1}^{m} O_{j_{k}}\right\rangle_{\rho}\right\}$. Moreover, each $\left\langle\otimes_{k=1}^{m} O_{j_{k}}\right\rangle_{\rho}$ will appear in some $\operatorname{tr} \rho B_{i}$ by the minimality of $\left\{\otimes_{k=1}^{m} O_{j_{k}}\right\}$. Hence $\rho$ 
can be written as

$$
\rho=\sum_{j_{1} j_{2} \cdots j_{m}}\left\langle\otimes_{k=1}^{m} O_{j_{k}}\right\rangle_{\rho} B_{j_{1} j_{2} \cdots j_{m}},
$$

where $B_{j_{1} j_{2} \cdots j_{m}}$ is a linear combination of $\left\{B_{i}\right\}$, which cannot be a zero matrix with respect to any basis of $V$ since $\left\{B_{i}\right\}$ is linearly independent. This proves the proposition.

Example with Broken Symmetry: Now let us present a concrete example to illustrate the results obtained above. We consider the case that $W$ is the state space of spin $1 / 2$ particle. Let $|\uparrow\rangle$ and $|\downarrow\rangle$ be the normalized eigenvectors of the Pauli operators $\sigma_{z}$ and take $V=V_{1} \otimes V_{2}, V_{1}=$ $W_{1} \otimes W_{2}, V_{2}=W_{3} \otimes \cdots \otimes W_{m}$.

We suppose the state $\rho$ to be measured possess the symmetry generated by $A=\otimes_{j=1}^{m} \sigma_{z}^{j}$, namely the symmetry of $\exp ($ it $A)$, where $m$ is even. Then according to Lemma 2, the reduced density operator $\tilde{\rho} \triangleq \operatorname{tr}_{2} \rho$ possesses the symmetry of $U=\exp \left(\right.$ it $\left.A_{1}\right)$ with $A_{1}=$ $\sigma_{z} \otimes \sigma_{z}$. Consequently, by Proposition 2, with respect to the basis $\{|\uparrow \uparrow\rangle,|\downarrow \downarrow\rangle,|\uparrow \downarrow\rangle,|\downarrow \uparrow\rangle\}$, the reduced density operator is of the 8-vertex form

$$
\tilde{\rho}=\left(\begin{array}{cccc}
r_{1} & z_{1} & & \\
z_{1}^{*} & r_{2} & & \\
& & r_{3} & z_{2} \\
& & z_{2}^{*} & r_{4}
\end{array}\right),
$$

where the $r_{i}$ 's are real numbers with the restriction $\sum_{i} r_{i}=1$ and $z_{i}^{*}$ stands for the complex conjugation of $z_{i}$. One can check that $\left\{1 \otimes 1,1 \otimes \sigma_{z}, \sigma_{z} \otimes 1, \sigma_{x} \otimes\right.$ $\left.\sigma_{x}, \sigma_{y} \otimes \sigma_{y}, \sigma_{z} \otimes \sigma_{z}, \sigma_{x} \otimes \sigma_{y}, \sigma_{y} \otimes \sigma_{x}\right\}$ is the unique reconstruction basis of $O_{U}$ associated with $\left\{\sigma_{i}\right\}$ and that $\left\{G_{00}^{\tilde{\rho}}, G_{03}^{\tilde{\rho}}, G_{30}^{\tilde{\rho}}, G_{11}^{\tilde{\rho}}, G_{22}^{\tilde{\rho}}, G_{33}^{\tilde{\rho}}, G_{12}^{\tilde{\rho}}, G_{21}^{\tilde{\rho}}\right\}$ is the cprresponding MCSQCF. The explicit expression of $\tilde{\rho}$ in terms of the MCSQCF is as follows: $r_{1}=\left(G_{33}^{\tilde{\rho}}+G_{30}^{\tilde{\rho}}+G_{03}^{\tilde{\rho}}+\right.$ 1) $/ 4, r_{2}=\left(G_{33}^{\tilde{\rho}}-G_{30}^{\tilde{\rho}}-G_{03}^{\tilde{\rho}}+1\right) / 4, r_{3}=\left(G_{30}^{\tilde{\rho}}-G_{33}^{\tilde{\rho}}-\right.$ $\left.G_{03}^{\tilde{\rho}}+1\right) / 4, \quad r_{4}=\left(G_{03}^{\tilde{\rho}}-G_{33}^{\tilde{\rho}}-G_{30}^{\tilde{\rho}}+1\right) / 4, z_{1}=\left(G_{11}^{\tilde{\rho}}-\right.$ $\left.G_{22}^{\tilde{\rho}}+i G_{12}^{\tilde{\rho}}+i G_{21}^{\tilde{\rho}}\right) / 4, z_{2}=\left(G_{11}^{\tilde{\rho}}+G_{22}^{\tilde{\rho}}+i G_{21}^{\tilde{\rho}}-i G_{12}^{\tilde{\rho}}\right) / 4$.

This example has a non-trivial connection with the Ising model with the Hamiltonian

$$
H=-J \sum_{j=1}^{m-1} \sigma_{z}^{j} \sigma_{z}^{j+1}+g \sum_{j=1}^{m} \sigma_{z}^{j}, J>0 .
$$

When the external field $g=0$, this model possesses the $S O(2) \otimes Z_{2}$ symmetry where $Z_{2}$ represents the rotations of all spins by 180 degrees about the x axis while $S O(2)$ represents the rotations of all spins by arbitrary degrees about the $\mathrm{z}$ axis. However, this system has two degenerate ferromagnetically ordered ground states $|\uparrow\rangle^{\otimes m}$ and $|\downarrow\rangle^{\otimes m}$, which break the discrete symmetry $Z_{2}$. Obviously, as a superposition of these two ground states, the cat state $|\psi\rangle=\alpha|\uparrow\rangle^{\otimes m}+\beta|\downarrow\rangle^{\otimes m}$ possess the symmetry of $\exp ($ it $A$ ) mentioned above since it is the eigenvector of $A=\otimes_{j=1}^{m} \sigma_{z}^{j}$ corresponding to the eigenvalue 1 , and then the unknown $\alpha$ and $\beta$ satisfying $|\alpha|^{2}+|\beta|^{2}=1$ can be determined by the above quantum state reconstruction method. When $g \neq 0$, the reduced density operator $\tilde{\rho}=\operatorname{tr}_{2} \rho$ derived from the ground state or the thermal equilibrium state(a function of hamiltonian)is of the 6vertex form for the existence of the total spin projection $\sum_{j} \sigma_{z}^{j}$. This result has appeared in many places, e.g., in the references [7, 8]

Concluding remarks : Based on the viewpoint of symmetry, we have proposed a quantum state reconstruction method for density operators or reduced density operators of the many body system. Our central idea is to utilize the MCSQCF determined by the symmetry of quantum states rather than the symmetry of the Hamiltonian. The present work might be regarded as a generalization of the "particle creation-annihilation expression" for the reduced density matrices of identical particles in Ref. 12], which reveals the essence of quantum condensation. Thus, we hope that the results in this letter can be directly applied to explore the quantum and classical critical phenomenon for various quantum many body systems.

This work is supported by the NSFC with grant Nos. 90203018,10474104 and 60433050 . It is also funded by the National Fundamental Research Program of China with Nos. 2001CB309310 and 2005CB724508.

* Electronic address: liuxf@pku.edu.cn

$\dagger$ Electronic address: suncp@itp.ac.cn URL: http://www.itp.ac.cn/ suncp

[1] W. Heisenberg, The physical principle of Quantum Theory, Univ. Chicago Press (Dover, NY, 1930); J. von Neumann, Mathematical Foundations of Quantum Mechanics, (Princeton Univ. Press, NJ, 1955).

[2] G. M. D'Ariano and H. P. Yuen, Phys. Rev. Lett. 76, 2832 (1996).

[3] W. K. Wootters and W. H. Zurek, Nature 299, 802 (1982).

[4] U. Fano, Rev. Mod. Phys. 29, 74 (1957), Sec. 6.

[5] D. T. Smithey, M. Beck, M. G. Raymer, and A. Faridani, Phys. Rev. Lett. 70, 1244 (1993);K. Vogel and H. Risken, Phys. Rev. A, 40, 2847 (1989); G. M. D'Ariano, C. Macchiavello and M. G. A. Paris, Phys. Rev. A 50, 4298 (1994).

[6] W. K. Wootters, Phys. Rev. Lett 80, 2245 (1998).

[7] K.M. O'Connor and W. K. Wootters, Phys. Rev. A 63, 052302 (2001).

[8] X. Wang and P. Zanardi, Phys. Lett. A 301, 1 (2002); X. Wang, Phys. Rev. A 66, 034302 (2002).

[9] G. Vidal, J. I. Latorre, E. Rico, and A. Kitaev,Phys. Rev. Lett. 90, 227902 (2003)

[10] S.-J. Gu, S.-S. Deng, Y.-Q. Li, and H.-Q. Lin, Phys. Rev. Lett. 93, 086402 (2004).

[11] X.F. Qian, T. Shi, Y. Li, Z. Song and C. P. Sun, Phys. Rev. A 72, 012333 (2005).

[12] C.N. Yang, Rev. Mod. Phys. 34, 694 (1962). 